Audiology

Neurotology
Audiol Neurotol 2020;25:72-78

DOI: $10.1159 / 000504286$
Received: August 15, 2019

Accepted after revision: October 21, 2019

Published online: December 11, 2019

\title{
Bilateral Vestibular Hypofunction in the Time of the Video Head Impulse Test
}

\author{
Nicolás Pérez-Fernández ${ }^{a}$ Laura Alvarez-Gomez ${ }^{b}$ Raquel Manrique-Huarte ${ }^{b}$ \\ ${ }^{a}$ Department of Otorhinolaryngology, Clínica Universidad de Navarra, Madrid, Spain; ${ }^{b}$ Department of \\ Otorhinolaryngology, Clínica Universidad de Navarra, Pamplona, Spain
}

\section{Keywords}

Vestibulo-ocular reflex · Nystagmus · Vestibule $\cdot$ Otoliths ·

Peripheral vestibular loss

\begin{abstract}
Objectives: Bilateral vestibulopathy is a clinical syndrome in which laboratory testing plays a crucial diagnostic role. We aimed to establish the frequency of detection of that finding in a tertiary level hospital considering the new methods of laboratory vestibular examination nowadays in use, with respect to the conventional caloric and rotatory chair test approaches. Design: Two similar time periods (5 years) were retrospectively evaluated, and the demographic, clinical data and test results from 4,576 patients were reviewed. In the first period, the diagnosis was based on caloric and rotatory chair tests and, in the second, on the video head impulse test. Results: Of the patients included, $3.77 \%$ in the first period and $4.58 \%$ in the second met the criteria for bilateral vestibular hypofunction; there was no significant difference between both periods. Conclusions: The functional vestibular evaluation to detect bilateral deficiency makes no significant difference to the number of patients diagnosed with a bilateral vestibulopathy. New diagnostic categories probably depend not only on the availability and accessibility of complete vestibular and visual-vestibular evaluation, but
\end{abstract}

also on recent advances in defining vestibular disorders. Bilateral vestibular hypofunction manifests with very different patterns. Progress in more detailed definition (clinical and laboratory) is needed, in particular when all 6 semicircular canals and both maculae are available for testing.

(c) 2019 The Author(s)

Published by S. Karger AG, Basel

\section{Introduction}

In vestibular testing, bilateral vestibular weakness or hypofunction $(\mathrm{BVH})$ refers to the finding of a lower than expected response after stimulation of both ears; it is usually incomplete or partial [Hain et al., 2013].

Patients usually consult the specialist because of chronic unsteadiness, and the following clinical data are characteristic in these patients: (1) unsteadiness is infrequently heralded by vertigo spells, (2) is progressive, (3) increases in darkness, and (4) is accompanied by visual blurring when walking (oscillopsia). The etiology (found to be diverse) dictates which of these symptoms prevails [Lucieer et al., 2016].

Its estimated prevalence in adults is $28 / 100,000$, and it has a major negative impact on the quality of life by limiting daily activities and increasing the risk of falls [Ward et al., 2013]. It accounts for $4 \%$ of the patients seen in ref-

\section{KARGER}

E-Mail karger@karger.com www.karger.com/aud
(C) 2019 The Author(s) Published by S. Karger AG, Basel

Karge

Open access

This article is licensed under the Creative Commons AttributionNonCommercial-NoDerivatives 4.0 International License (CC BYNC-ND) (http://www.karger.com/Services/OpenAccessLicense). Usage and distribution for commercial purposes as well as any distribution of modified material requires written permission.
Nicolas Pérez-Fernández

Department of Otorhinolaryngology, Clinica Universidad de Navarra Calle del Marquesado de Santa Marta 1

ES-28027 Madrid (Spain)

E-Mail nperezfer@unav.es 
erence centers for vestibular evaluation [Zingler et al., 2009].

Vestibular rehabilitation, a well-established method of treatment for patients diagnosed with BVH, gives good results in only some of the different outcome measures evaluated (dynamic visual acuity, gait speed and dynamic gait index), but no patient has all these results, unlike cases with unilateral vestibulopathy following the same treatment [Herdman et al., 2015]. New methods of treatment (the vestibular implant) open a line of research for patients with this disorder.

When BVH is suspected, after bedside vestibular examination, laboratory testing is mandatory in order to correctly characterize the degree of dysfunction. This is usually performed with the caloric test and rotatory chair testing. When the caloric response from both ears is found to be below the normal expected response, it is imperative to: (1) rule out technical artifacts or erroneous results due to lack of alertness and (2) to confirm the finding. Two tests are used to achieve these objectives: the ice water caloric test [Batuecas-Caletrío et al., 2009] and the rotatory chair test [Honrubia et al., 1985].

The video head impulse test (vHIT) is a test of the angular vestibulo-ocular reflex (VOR), not of nystagmus. It has recently been introduced and allows independent testing of the function of the vestibular receptors in the ampullae of each of the semicircular canals (SC) [Weber et al., 2009; Curthoys, 2012]. Its place in the evaluation of patients with dizziness is midway between bedside and laboratory, and its use as the first test has been recommended in the view that it can optimize diagnosis [EzaNuñez et al., 2016; van Esch et al., 2016].

In this study, we were interested in reviewing the frequency of diagnoses of BVH in the vestibular laboratory since the introduction of the vHIT testing of patients; we therefore reviewed the clinical history of all the patients with BVH to analyze their etiologies.

\section{Materials and Methods}

\section{Patients and Periods of Study}

This is a retrospective study. Patients included were those seen at our institution and likely to be tested in our vestibular laboratory. The patients were divided on the basis of two different time periods: series 1 (caloric testing, CAL, period) between September 1, 2006, and August 31, 2011, and series 2 (vHIT period) between September 1, 2012, and August 31, 2017. In the CAL period, all were tested with the caloric test (unless patients had chronic otitis media) and the rotatory chair test, and in the vHIT period all subjects included underwent vHIT testing. The protocol for this study was approved by the institutional review board at our hospital; previously all patients had signed informed consent for the testing. The research was in accordance with the World Medical Association Declaration of Helsinki.

Tests Performed and Definition of BVH

CAL Period

Caloric Test. The Fitzgerald and Hallpike alternate bithermal caloric test (with water) was used which records eye movements by means of a video-based system (Ulmer VNG, v. 1.4, SYNAPSIS ${ }^{\circledR}$, Marseille, France). The maximum slow phase velocity of nystagmus was calculated after each irrigation.

Rotatory Chair Test. This was performed using a CHARTR ${ }^{\circledR}$ RVT system (ICS Medical Corporation, Schaumburg, IL, USA). The rotatory chair is housed in an enclosure in order to perform the test in the dark, and the head is restrained and positioned so that both horizontal canals are close to the plane of stimulus (i.e., at the gravitational horizontal). In the impulsive rotational test, the patient was subjected to velocity steps to the right and left. The velocity steps involved the patient undergoing an angular acceleration of $100^{\circ} \mathrm{s}^{-1}$ for $1 \mathrm{~s}$, rotation at a constant velocity for $60 \mathrm{~s}$, and finally a deceleration to $0^{\circ} \mathrm{s}^{-1}$ within $1 \mathrm{~s}$, which gives 2 time constant (TC) values: clockwise and counterclockwise, respectively. This procedure was performed 3 times, and the TC was averaged $\left(\mathrm{TC}_{\mathrm{ave}}\right)$. In the sinusoidal harmonic acceleration test (SHA), the individual was subjected to sinusoidal oscillation about a yaw axis at various frequencies $(0.01,0.02,0.04,0.08,0.16,0.32$ and 0.64 $\mathrm{Hz}$ ), with a peak angular velocity of $50^{\circ} \mathrm{s}^{-1}$. From the chair velocity and slow-phase eye velocity, 2 parameters of the VOR were calculated: phase and gain.

Bilateral Vestibular Hypofunction. The response in the following three tests had to be abnormal: (1) in the alternate bithermal caloric test, reflectivity $<15^{\circ} \mathrm{s}^{-1} ;(2)$ in the impulsive test, the $\mathrm{TC}_{\text {ave }}$ $<8 \mathrm{~s}$, and (3) in the SHA test, gain and phase had to be significantly lower than normal in at least three consecutive frequencies. The ice water test was performed in all these patients.

\section{vHIT Period}

vHIT. It was performed with a GN Otometrics system. For this test the patient wears a pair of lightweight, tightly fitting goggles on which is mounted a small video camera and a mirror that reflects the image of the patient's right eye into the camera. The eye is illuminated by a low-level infra-red light-emitting diode. A small sensor on the goggles measures the head movement. The whole goggle system is secured tightly to the head to minimize goggle slippage. Calibration is performed, and the procedure of vestibuloocular testing is initiated. Horizontal SC (right and left) are first tested: the clinician asks the patient to keep staring at an earthfixed target $1 \mathrm{~m}$ in front and gives the patient brief, abrupt, head rotations through a small angle (about $10-20^{\circ}$ ), unpredictably turning to the left or right on each trial. At the end of each head turn, the head velocity stimulus and eye velocity response are displayed simultaneously on the screen. In a full test, 20 impulses are delivered randomly in each direction. The first pair of vertical canals is then evaluated: the patient's head is rotated $30^{\circ}$ to the right and while staring at the same earth-fixed target as before, but now out of the corner of his/her eye. Brief, abrupt, forward and backward head impulses are then given that allow stimulation of the left anterior SC and the right posterior SC, respectively. After a full test of 20 impulses in each direction, the second pair of vertical canals is evaluated: for this, the patient's head is rotated $30^{\circ}$ to the left 


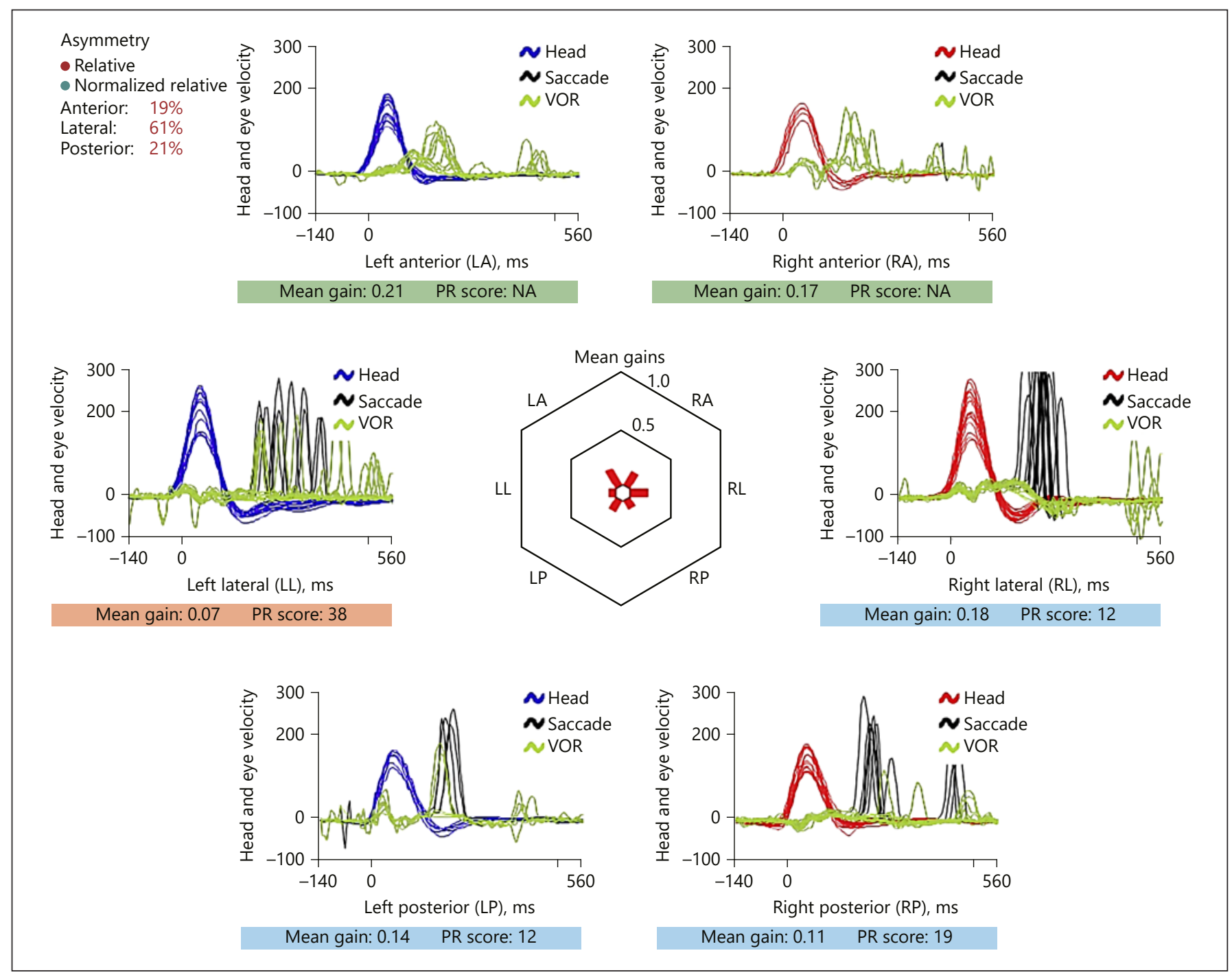

Fig. 1. vHIT result in a patient (male, 63 years old) with a bilateral vestibulopathy; the cause is unknown. He was seen for chronic unsteadiness that had progressively worsened over the previous 2 years. Symmetric high-frequency hearing loss in accordance with age. Vestibular evoked myogenic potentials (for bone-conducted stimulus) were bilaterally absent. Responses for all 6 semicircular canals were below normal and expected limits for age. The PR score is a measure of the temporal agreement of refixation saccades in successive head impulses: isochronic saccades give a lower value than heterochronic ones, which provide a higher score (up to 100). while staring at the earth-fixed target. Now, forward head impulses stimulate the right anterior SC and backward the left posterior SC. A full test of 20 impulses in each direction is performed. The parameter evaluated was the VOR mean gain (ratio of eye velocity to head velocity) that is obtained from the gain value after each of the impulses performed and is automatically provided for rightward and leftward horizontal head impulses, left anterior and right posterior head impulses, and the right anterior and left posterior head impulses.

Bilateral Vestibular Hypofunction. The response from the 6 SC had to be below normal established limits for age [Matiño-Soler et al., 2015; McGarvie et al., 2015] as shown in Figure 1.

\section{Statistics}

All data were stored and analyzed in an SPSS file version 19.0 (SPSS Inc.; Chicago, IL, USA). All tests were two-tailed, and $p$ values $<0.05$ were considered significant. A $\chi^{2}$ test was performed to assess the differences in $\mathrm{BVH}$ detection. Nonparametric tests were used to evaluate differences between patients in the two series here considered (Mann-Whitney U test), and Spearman's $\rho$ was used to detect correlations between measures within each series.
Pérez-Fernández/Alvarez-Gomez/ Manrique-Huarte 


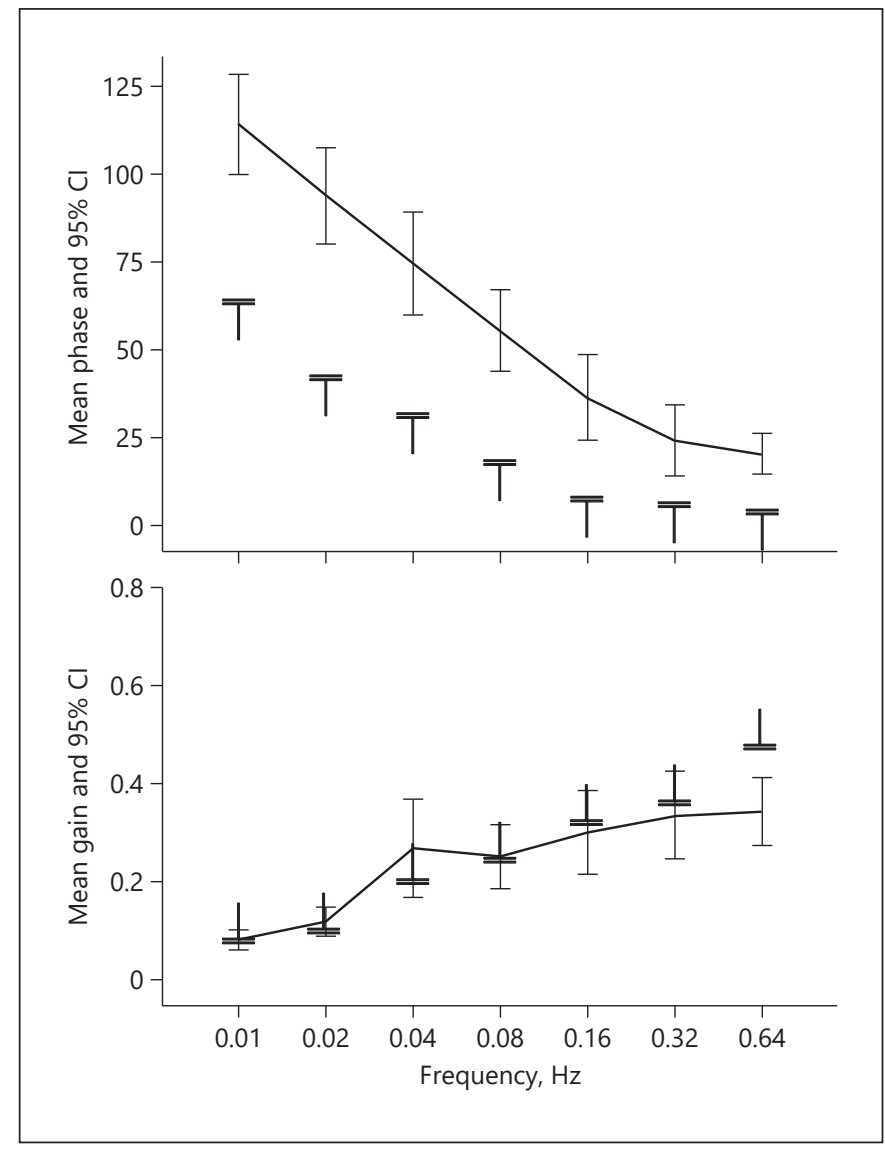

Fig. 2. Phase and gain of VOR in the sinusoidal harmonic acceleration (SHA) test. Results are means and 95\% confidence intervals and are shown by frequency of stimulation. T labels indicate the upper and lower limits of normal data in our lab for phase lag and gain, respectively, in the SHA test.

\section{Results}

In this study, we included the charts from 4,576 patients. 1,961 patients belonged to the CAL period (series 1 ) and 2,615 patients to the vHIT period (series 2). In the first group 74 (3.77\%) were diagnosed as having BVH, and in the second, 120 (4.58\%). Although the percentage of bilateral cases seems to have increased, the $\chi^{2}$ statistic is $1.64(p=0.2)$.

\section{Frequency of the Finding and Clinical Characteristics}

In Table 1 we present the main demographic and clinical data for both series of patients. As a whole, differences between the CAL series and the vHIT series were seen only in age, as in series 2 the patients were slightly older (Mann-Whitney U, $p=0.035$ ). Although in both

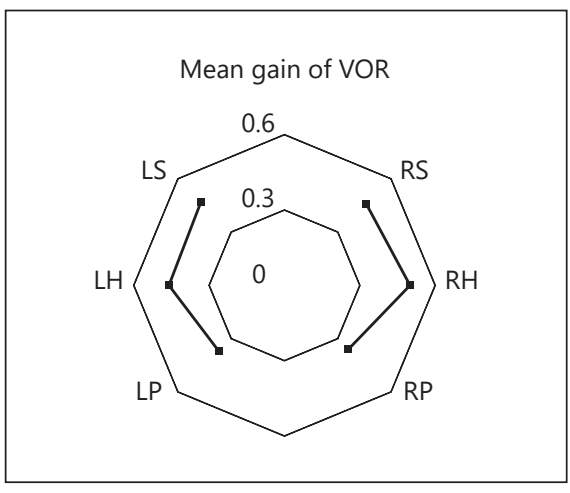

Fig. 3. Representation of the mean gain for each of the canals tested. RS, right superior; RH, right horizontal; RP, right posterior; LP, left posterior; LH, left horizontal; LS, left superior.

Table 1. Clinical and demographic patient data in both series

\begin{tabular}{lll}
\hline & $\begin{array}{l}\text { Series 1 } \\
\text { (CAL period) }\end{array}$ & $\begin{array}{l}\text { Series 2 } \\
\text { (vHIT period) }\end{array}$ \\
\hline $\begin{array}{ll}\text { Patients with BVH, \% } \\
\text { Male:female, } n\end{array}$ & 3.77 & 4.58 \\
Age (95\% CI), years & $46: 28$ & $57: 63$ \\
$\begin{array}{l}\text { Oscillopsia, \% } \\
\text { Unsteadiness of gait worse in } \\
\quad \text { darkness, \% }\end{array}$ & $57(53-61)$ & $66(51-71)$ \\
$\begin{array}{l}\text { Migraine, \% } \\
\text { Dizziness Handicap Inventory }\end{array}$ & 19 & 16 \\
$\quad$ score (mean \pm SD) & 73 & 63 \\
\hline
\end{tabular}

series the Dizziness Handicap Inventory score increased with age, both were not correlated (Spearman $\rho, p=0.144$ for the CAL series, and Spearman $\rho, p=0.76$ for the vHIT series).

\section{Results of Tests}

\section{CAL Period}

The caloric test was carried out on $69 / 74$ patients. At diagnosis: (1) mean reflectivity in the caloric test was $5.6^{\circ}$ $\mathrm{s}^{-1}\left(95 \% \mathrm{CI}: 4.4-6.8^{\circ} \mathrm{s}^{-1}\right)$; (2) the mean $\mathrm{TC}_{\text {ave }}$ of the VOR was $3.2 \mathrm{~s}$ (95\% CI: $2.3-4.3 \mathrm{~s}$ ); and (3) the mean gain and phase of the VOR as assessed in the SHA test are shown in Figure 2. In 5 patients with bilateral chronic otitis media and clinical suspicion of $\mathrm{BVH}$, on whom we could not carry out the caloric test, the mean $\mathrm{TC}_{\mathrm{ave}}$ of the VOR was $4 \mathrm{~s}$ (95\% CI: 1.9-6.3); in all of them the SHA testing criteria were also fulfilled. There was no response to ice water. 
Fig. 4. Distribution of patients diagnosed with BVH in this study according to etiology. Series 1 ( $n=74$ patients) is in white and series 2 ( $n=120$ patients) in grey.

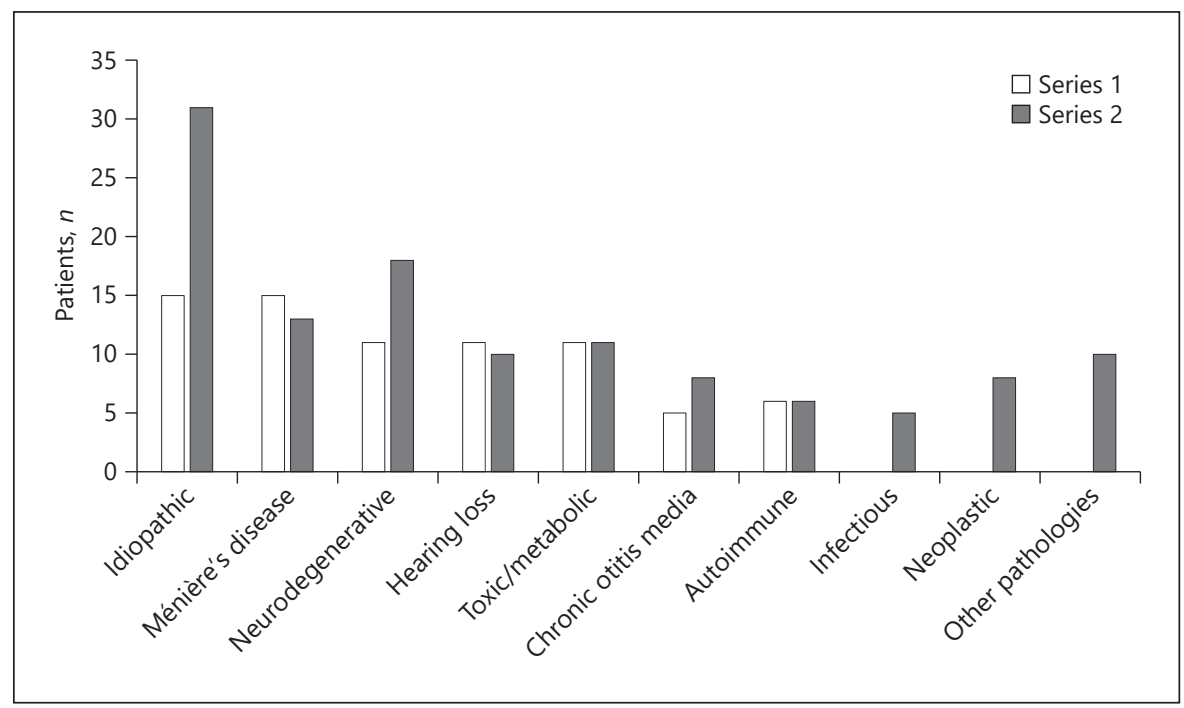

\section{vHIT Period}

The mean gain of VOR for each of the canals tested is shown as a polar plot in Figure 3.

\section{Etiologies Identified in Patients with BVH}

Final diagnoses are shown in Figure 4. We tried to use and match our classification to that recently used for the classification of patients with bilateral vestibulopathy [Lucieer et al., 2016]. In general, idiopathic BVH is the most frequent category (25.7\%), and its frequency is very close to what others have found. Ménière's disease is the second $(17 \%)$ in frequency and first of the nonidiopathic types, which is probably due to the frequency of that diagnosis at our hospital; these patients were classified according to current guidelines at the time of evaluation [Committee on Hearing and Equilibrium guidelines for the diagnosis and evaluation of therapy in Ménière's disease, $1995]$ as definite in both ears in 18 patients (54.5\%), definite in one ear and probable in the other in 9 patients $(27.2 \%)$, and definite in one ear and another diagnosis (inducing unilateral vestibulopathy) in the second ear in 6 patients (18.3\%). In the neurodegenerative category, we include our 5 definite cases of CANVAS (cerebellar ataxia, neuropathy, vestibular areflexia). In the hearing loss category, there are 12 patients who had received a cochlear implant unilaterally and, either before or after implantation, were assessed for bilateral vestibulopathy. In the toxic/ metabolic category we include mainly patients treated with ototoxic medication. Three categories were not in the CAL period patients: "infectious" in which 3 patients were diagnosed with bilateral sequential vestibular neuritis and 2 as having sequelae of meningitis with associated hearing loss; "neoplastic" with 2 cases of neurofibromatosis type 2 and 4 cases of metastatic malignant disease not related to the temporal bone or VIIIth nerve, and 2 cases considered as probable paraneoplastic syndrome [Greene et al., 2015]. Some patients without common characteristics were classified in a category reported as "other": these were mainly mixed cases, such as 1 case of Pendred syndrome and inner ear concussion, 2 cases with vestibular migraine, 3 cases with posttraumatic damage, and 4 cases of "presbyvertigo."

\section{Discussion/Conclusion}

In this study we have shown that vHIT testing among patients complaining of unsteadiness does not increase the incidence of BVH reporting in our laboratory. In our department and during the period of time included in this study, the number of doctors attending patients with vestibular disorders was constant and test availability did not change. In both periods of time, BVH detection was based mainly on clinical history and bedside testing, relying particularly on the bedside HIT, dynamic visual acuity and Romberg tests [Petersen et al., 2013].

One limitation to this first conclusion concerns the origin of our study and data. This is a retrospective evaluation in which data were taken from the vestibular laboratory files. It was not possible to assess how many patients were suspected of having BVH and whose diagnosis was, or was not, confirmed in the laboratory, but it has been reported that the sensitivity of the bedside HIT to detect 
BVH is $84 \%$ while the specificity is $82 \%$ [Schubert et al., 2004]. Given the need to confirm any suspicion with laboratory tests, our results could be biased, given the argument that the CAL period may include patients with a less disabling condition which allows them to proceed through caloric and rotatory testing, but excludes some others with a more serious condition (in an attempt to avoid the detrimental effects of prolonged vestibular testing) who did not undergo testing. We do not think that is the case given that the reported mean Dizziness Handicap Inventory score for both CAL and vHIT periods showed no significant differences. In this regard we acknowledge that the ideal workup in patients with suspected BVH by either method (CAL or vHIT) should have to be confirmed in the other; the existence of differences in results between both techniques is a well-known finding in patients with hydropic inner ear disease. That, we agree, should be the best protocol for evaluation and the analysis of results. Another limitation is that we have not evaluated a subgroup of patients with only horizontal canal deficiency, or the most common horizontal and posterior canal. This would increase the numbers in vHIT detection. This is currently under way together with the assessment in the caloric test given that those are the canals exactly evaluated in the later testing.

Regarding the criteria used to define when a vHIT is abnormal, there are two different issues to which attention must be paid. One is the response itself in which some discrepancies are found, as some researchers use a fixed amount of gain as the limit for a normal response: 0.8 for the horizontals [Moon et al., 2017] and 0.7 for the verticals [Tarnutzer et al., 2016]. We decided to use data according to age as this represents a more realistic view of the evaluation given the mean age of the expected patients in whom we were to diagnose a BVH. Some degree of discrepancy between both criteria could be seen in the case of the verticals (gain $<0.7$ but still normal for age) due to the fact that variability of gain is greater in those canals than in the horizontals because of a more rapid dropoff in VOR gain as head velocity increases [McGarvie et al., 2015]. A second issue regarding another criterion used to define $\mathrm{BVH}$ with the vHIT is the existence of observable refixation (covert and overt) saccades; these were seen in all our patients.

When defining $\mathrm{BVH}$ at present, another relevant issue is which canals need to be deficient [Tarnutzer et al., 2018]. Recently it has been shown that both anterior canals can be spared (keeping a normal gain and no refixation saccades upon vHIT testing) in aminoglycoside-associated $\mathrm{BVH}$, in $\mathrm{BVH}$ of unknown cause or due to Ménière's disease. On the contrary, a BVH can occur in certain central diseases with

Bilateral Vestibulopathy: Caloric versus vHIT involvement of only the horizontal canals, sparing the verticals, as occurs in acute Wernicke's encephalopathy [Akdal et al., 2016]. At this point, and without trying to identify a given pattern of canal sparing, we decided to define $\mathrm{BVH}$ based on clinical suspicion and when the 6 SC tested provide an abnormal response in gain.

The place of vestibular evoked myogenic potential testing has not been evaluated in depth, though it has been shown that saccular function is much better preserved than canal function when evaluated with the caloric test [Zingler et al., 2008]; also, in that modality of canal function evaluation, there is a correlation of results with utricular function [Agrawal et al., 2013].

Regarding etiology the diagnostic categories remain stable with some differences. It is particularly interesting for this syndrome to construct a framework for diagnosis, as has recently been stated [Strupp et al., 2017]; however, the number of different diagnoses given is very extensive and will probably have to include mixed and very unusual disorders.

We agree with previous authors who state that "for the future diagnostic process of BVH, we would strongly suggest performing a vHIT instead of a manual HIT" [Lucieer et al., 2016; Strupp et al., 2017]. However, in cases with doubtful responses, it is recommended to add the CAL period criteria in the diagnostic workup. The ability to test at the bedside (as the vHIT is used in common practice) allows us to receive immediate information about the VOR for all patients without almost any limitation. In its development two new tests could be implemented to increase the differential diagnostic ability of the vHIT in patients with BVH. The first one is the VOR suppression, with the newly presented suppression head impulse paradigm [Shen et al., 2016] which is helpful: (1) to better measure VOR gain and residual vestibular function, in particular when in the common HIT gain is very much reduced and with covert saccades interfering with the registry, and (2) to detect anticompensatory saccades indicating a procedure for VOR adaptation to minimize retinal slip and probably oscillopsia in chronic patients. The second test is the visual-vestibular interaction test, which is of major interest when at first glance it is imperative to define whether a peripheral or central deficiency is most likely [Rey-Martinez et al., 2018].

In conclusion, BVH detection has not increased since the introduction of vHIT though the protocol of evaluation has become more straightforward, with much less discomfort for the patient, if we take into consideration the time needed for the combined caloric and rotatory chair tests and their residual effects.

Audiol Neurotol 2020;25:72-78

DOI: $10.1159 / 000504286$ 


\section{Acknowledgment}

The authors wish to acknowledge the effort of our technicians in the vestibular laboratory: Mari Cruz Betelu and Patricia Rodriguez.

\section{Disclosure Statement}

The authors disclose no possible conflicts of interest. There was no external support or financial involvement in the study.

\section{References}

Agrawal Y, Bremova T, Kremmyda O, Strupp M. Semicircular canal, saccular and utricular function in patients with bilateral vestibulopathy: analysis based on etiology. J Neurol. 2013 Mar;260(3):876-83.

AkdalG, MacDougall HG, Chen L, Tanriverdizade T, Yiğitaslan O, Halmagyi GM. Selective impairment of horizontal vestibulo-ocular reflexes in acute Wernicke's encephalopathy. J Neurol Sci. 2016 Jun;365:167-8.

Batuecas-Caletrio A, Montes-Jovellar L, BoleasAguirre MS, Perez-Fernandez N. The ice-water caloric test. Acta Otolaryngol. 2009 Dec; 129(12):1414-9.

Committee on Hearing and Equilibrium guidelines for the diagnosis and evaluation of therapy in Menière's disease. American Academy of Otolaryngology-Head and Neck Foundation, Inc. Otolaryngol Head Neck Surg. 1995 Sep;113(3):181-5.

Curthoys IS. The interpretation of clinical tests of peripheral vestibular function. Laryngoscope. 2012 Jun;122(6):1342-52.

Eza-Nuñez P, Fariñas-Alvarez C, Fernandez NP. Comparison of three diagnostic tests in detecting vestibular deficit in patients with peripheral vestibulopathy. J Laryngol Otol. 2016 Feb;130(2):145-50.

Greene JJ, Keefe MW, Harris JP, Matsuoka AJ. Paraneoplastic syndrome: a masquerade of autoimmune inner ear disease. Otol Neurotol. 2015 Jan;36(1):e3-10.

Hain TC, Cherchi M, Yacovino DA. Bilateral vestibular loss. Semin Neurol. 2013 Jul;33(3): 195-203.

Herdman SJ, Hall CD, Maloney B, Knight S, Ebert $\mathrm{M}$, Lowe J. Variables associated with outcome in patients with bilateral vestibular hypofunction: preliminary study. J Vestib Res. 2015; 25(3-4):185-94.
Honrubia V, Marco J, Andrews I, Minser K, Yee $\mathrm{RD}$, Baloh RW. Vestibulo-ocular reflexes in peripheral labyrinthine lesions: III. Bilateral dysfunction. Am J Otolaryngol. 1985 SepOct;6(5):342-52.

Lucieer F, Vonk P, Guinand N, Stokroos R, Kingma $H$, van de Berg R. Bilateral vestibular hypofunction: insights in etiologies, clinical subtypes, and diagnostics. Front Neurol. 2016 Mar; 7:26.

Matiño-Soler E, Esteller-More E, Martin-Sanchez JC, Martinez-Sanchez JM, Perez-Fernandez N. Normative data on angular vestibulo-ocular responses in the yaw axis measured using the video head impulse test. Otol Neurotol. 2015 Mar;36(3):466-71.

McGarvie LA, MacDougall HG, Halmagyi GM, Burgess AM, Weber KP, Curthoys IS. The video head impulse test (vHIT) of semicircular canal function. Age-dependent normative values of VOR gain in healthy subjects. Front Neurol. 2015 Jul;6:154.

Moon M, Chang SO, Kim MB. Diverse clinical and laboratory manifestations of bilateral vestibulopathy. Laryngoscope. 2017 Jan; 127(1):E42-9.

Petersen JA, Straumann D, Weber KP. Clinical diagnosis of bilateral vestibular loss: three simple bedside tests. Ther Adv Neurol Disorder. 2013 Jan;6(1):41-5.

Rey-Martinez J, Batuecas-Caletrio A, Matiño E, Trinidad-Ruiz G, Altuna X, Perez-Fernandez N. Mathematical methods for measuring the visually enhanced vestibulo-ocular reflex and preliminary results from healthy subjects and patient groups. Front Neurol. 2018 Feb 12;9:69.

Schubert MC, Tusa RJ, Grine LE, Herdman SJ. Optimizing the sensitivity of the head thrust test for identifying vestibular hypofunction. Phys Ther. 2004 Feb;84(2):151-8.
Shen Q, Magnani C, Sterkers O, Lamas G, Vidal PP, Sadoun J, et al. Saccadic velocity in the new suppression head impulse test: a new indicator of horizontal vestibular canal paresis and of vestibular compensation. Front Neurol. 2016 Sep;7:160.

Strupp M, Kim JS, Murofushi T, Straumann D, Jen JC, Rosengren SM, et al. Bilateral vestibulopathy: Diagnostic criteria Consensus document of the Classification Committee of the Bárány Society. J Vestib Res. 2017;27(4):17789.

Tarnutzer AA, Bockisch CJ, Buffone E, Weber KP. Hierarchical cluster analysis of semicircular canal and otolith deficits in bilateral vestibulopathy. Front Neurol. 2018 Apr 10;9:244.

Tarnutzer AA, Bockisch CJ, Buffone E, Weiler S, Bachmann LM, Weber KP. Disease-specific sparing of the anterior semicircular canals in bilateral vestibulopathy. Clin Neurophysiol. 2016 Aug;127(8):2791-801.

van Esch BF, Nobel-Hoff GE, van Benthem PP, van der Zaag-Loonen HJ, Bruintjes TD. Determining vestibular hypofunction: start with the video-head impulse test. Eur Arch Otorhinolaryngol. 2016 Nov;273(11):3733-9.

Ward BK, Agrawal Y, Hoffman HJ, Carey JP, Della Santina CC. Prevalence and impact of bilateral vestibular hypofunction: results from the 2008 US National Health Interview Survey. JAMA Otolaryngol Head Neck Surg. 2013 Aug;139(8):803-10.

Weber KP, MacDougall HG, Halmagyi GM, Curthoys IS. Impulsive testing of semicircularcanal function using video-oculography. Ann N Y Acad Sci. 2009 May;1164(1):486-91.

Zingler VC, Weintz E, Jahn K, Bötzel K, Wagner J, Huppert D, et al. Saccular function less affected than canal function in bilateral vestibulopathy. J Neurol. 2008 Sep;255(9):1332-6.

Zingler VC, Weintz E, Jahn K, Huppert D, Cnyrim $\mathrm{C}$, Brandt T, et al. Causative factors, epidemiology, and follow-up of bilateral vestibulopathy. Ann N Y Acad Sci. 2009 May; 1164(1):505-8. 